\title{
Plonger : Risque, désir, mobilité et création au féminin chez Georgette LeBlanc
}

\author{
Joëlle PAPILLON, Université McMaster
}

Si Georgette LeBlanc avait mis au premier plan l'exploration de la subjectivité féminine dans son premier roman poétique Alma (2006), celle-ci avait ensuite été mise en veilleuse dans les deux suivants, Amédé (2010) et Prudent (2013), centrés sur des personnages masculins et reléguant l'expérience féminine du monde au second plan. Avec Le grand feu, roman poétique paru au printemps 2016, LeBlanc renoue avec des personnages féminins forts, notamment ceux de Cécile Murat, une jeune poète orpheline, et de Marguerite Thériault, une redoutable femme d'affaires. Toutes deux avaient fait leur première apparition dans la nouvelle poétique « $\mathrm{S}(\mathrm{H})$ hhhhhhh $»^{1}$, texte qui se présente comme un accompagnement à la trame narrative du Grand feu. Bien qu'elles soient des personnages très différents du point de vue de l'âge, du statut, de leur personnalité et de leur occupation, Cécile et Marguerite ont en commun de vivre une métamorphose lors de la soirée du Tyme, une grande fête qui se transformera en incendie. C'est en décidant de plonger, de prendre des risques, que chacune se choisira en disant « oui » au désir et aux mots, optant pour la voie de la régénération (Grand feu, 7) par le feu et l'eau.

Par elle-même, la nouvelle « $\mathrm{S}(\mathrm{H}) \mathrm{hhhhhhh»} \mathrm{demeure} \mathrm{assez} \mathrm{difficile} \mathrm{à} \mathrm{décrypter.} \mathrm{Lors}$ d'une fête de village, une jeune femme plonge dans un lac dans lequel elle est presque certaine de mourir. L'œuvre poétique suit Cécile alors qu'elle court vers le lac et s'élance sous l'eau, où elle a diverses visions qui se rapportent principalement à elle-même, mais aussi à Marguerite qu'elle voit sous un jour différent une fois qu'elle a quitté la surface. Les marqueurs spatiotemporels sont flous, voire contradictoires, évoquant le monde du rêve et du cinéma. La parution du Grand feu offre des clés d'interprétation importantes, plaçant l'action des deux récits dans un village de Kespukwitk (la Baie Sainte-Marie, en Nouvelle-Écosse) au dix-neuvième siècle, et complétant le portrait des deux personnages principaux. Pour célébrer la Sainte-Cécile, Marguerite organise une grande fête, le Tyme ; toute la communauté se rassemble chez elle, dans le bois à la Compound ${ }^{2}$, sur le bord d'un lac. La Dame est nerveuse parce qu'elle veut séduire l'homme qu'elle aime, le Capitaine Doucet, et cherche à créer la fête la plus mémorable qui soit. L'urgence de son désir se heurte à l'image obsédante de la Mary Celeste, un bateau fantôme qui hante l'imagination des villageois ; fascinée et angoissée par les récits qui circulent à son sujet, 
Marguerite imagine le bateau errant comme une maison vide avec une famille disparue - et notamment une petite fille. De façon obscure, la figure de cette enfant perdue est ce qui incite Marguerite à répondre enfin à l'appel de l'amour. Le soir de la fête est également un moment important pour Cécile, une des jeunes employées de Marguerite, qui doit quitter Kespukwitk le lendemain en compagnie de son oncle Joachim Murat qui la ramènera en Europe pour obéir, selon lui, aux volontés du père de Cécile, mort en mer. Cécile ne veut pas quitter Kespukwitk ni son amoureux Jean-Baptiste, mais elle se sent obligée de le faire, obéissant aux normes sociales de son époque. Toutefois, avant de partir, elle a décidé de se jeter dans le lac lors de la fête, une tradition locale à laquelle se plient les poètes et les musiciens. Sûre de mourir, elle se lance tout de même, plonge, et devient ainsi écrivaine. Le grand feu annoncé dès le titre du roman poétique ravage le domaine de Marguerite et les bois alentour, mais épargne les deux femmes ; l'incendie sert surtout à souligner l'intensité de la métamorphose des personnages principaux.

La différence de statut entre Cécile et Marguerite est frappante: en ouverture à « $\mathrm{S}(\mathrm{H}) \mathrm{hhhhhhh} »$, la première est surnommée «la p'tite Murat» (103) - ce qui la renvoie à son jeune âge mais aussi à la fragilité de sa position, elle qui est désormais sans père - tandis que la seconde est généralement nommée «la Dame », une marque de respect pour «celle qui construit Kespukwitk [...] et la rend prospère » (103). Marguerite est admirée et respectée pour son rôle de «fondatrice » de la communauté alors même que Kespukwitk existait bien avant son arrivée ${ }^{3}$; si elle la «construit », c'est en y apportant sa fortune et en y construisant des lieux de socialisation (le Casino, la Cantine, la Compound) qui transforment le village acadien en destination recherchée (Grand feu, 35), en «centre du monde » ${ }^{4}$. Marguerite est donc avant tout une entrepreneure, quelqu'un qui sait faire de l'argent mais qui sait aussi en faire bénéficier sa communauté - par exemple en organisant la grande fête du Tyme à laquelle tout le monde est convié.

\section{S'ouvrir au désir}

Digne héritière $\mathrm{d}^{\mathrm{A}} \mathrm{Alma}^{5}$ - la première hérö̈ne de LeBlanc -, Cécile est posée en rapport positif avec son corps dès son apparition dans Le grand feu :

Cécile sentait les braises de la nuit jusqu'à dans son corps, son temple à elle les voyait dans le ciel, le matin, au bord du lac en quittant la camp à Jean-Baptiste, [...] 
vite, retourner au logis sans réveiller ses parents (19)

Rentrant de chez son amoureux au petit matin, Cécile est présentée comme désirante et désirée, ce qui s'exprime par une première métaphore liée au feu (« les braises de la nuit ») qui indique à la fois son désir pour Jean-Baptiste et l'empreinte chaude que le territoire laisse sur elle. Voir l'aube lui procure une forte sensation physique, comme si elle était traversée de façon sensuelle par le paysage. Ainsi, Cécile est pleinement dans son corps et dans le territoire, elle habite le territoire autant que le territoire l'habite. Pour cette raison, c'est non seulement l'idée de devoir quitter Jean-Baptiste qui lui brise le cœur, mais aussi celle de devoir renoncer à la beauté singulière de Kespukwitk, présenté comme un « royaume » (19), une «terre sacrée » (34), un «Paradis » où «la fortune se trouvait $/$ si tu savais djetter ${ }^{6} »(15)$. De plus, son histoire avec Jean-Baptiste lui apparaît comme « une romance, un conte » (19), qu'elle veut vivre et écrire (20). Pour elle, le désir, l'amour, le territoire et l'écriture se rejoignent ainsi en un continuum fragile, menacé par la conviction de son oncle Joachim Murat, « Roi de Naples » ${ }^{7}$ (11), qu'il sait ce qui est mieux pour elle : la transplanter en Europe et faire d'elle une dame à travers de belles manières, un bon mariage et une belle langue - c'est-à-dire en la recadrant selon, d'une part, les normes linguistiques françaises ${ }^{8}$ et, $\mathrm{d}^{\prime}$ autre part, les normes de la féminité distinguée qui exigent une séparation des sphères masculines et féminines ainsi que l'abandon du travail.

L'autre personnage central des deux œuvres à l'étude, Marguerite, n'a en apparence pas le même problème que Cécile : en raison de sa puissance au sein de la communauté, personne n'oserait prétendre faire des choix à sa place et la contraindre à quoi que ce soit. Enfant de la Déportation née en exil (38), Marguerite est loin d'être dans la victimisation ; au contraire, elle se démarque par sa résilience, son indépendance, sa détermination et son intrépidité en affaires : pour elle, le «glass ceiling fracassé » crée « des milliers de diamants » $(« \mathrm{~S}(\mathrm{H}) \mathrm{hhhhhhh} », 108)$ qui s'étalent à ses pieds, et LeBlanc insiste sur son statut d'exception à Kespukwitk. Pourtant, jusque-là, Marguerite a toujours gardé les hommes à une certaine distance; non par idéal vertueux - elle qui a bâti sa fortune sur le jeu («c'était point de sa faute si la débauche existait / c'était tout ce qu'elle avait connu / tout ce qu'elle savait de vrai » [Grand feu, 60]) - mais par peur de l'enfantement. Alors qu'elle est en train de se faire masser ${ }^{9}$ avant la fête, elle se perd dans ses pensées, hésitant à s'ouvrir, craignant de se perdre :

la Dame sentait les mains sur son corps pousser, creuser, les noucles ${ }^{10}$ des années le refus, son refus 
malgré les histoires que ses curves faisiont raconter

la Dame avait vu trop de femmes se faire massacrer

le corps, les tripes, accouchements prématurés,

tromperies, abandons, vulnérabilité

sa propre mère était morte, l'avait quittée

ou c'était-il elle qui l'avait tuée

en naissant?

la Dame la voyait là, couchée

ce qu'on lui avait raconté

fallait garder ses distances

il y avait des conséquences à l'entrée

on lui avait fait trop mal pour croire aux contes de fées

la masseuse poussait, tordait comme le Doucet tentait

s'essayait, quand ce qu'il pouvait, l'assurait

il serait là, sagefemmes, les meilleures, elle serait entourée

il avait la besonne ${ }^{11}$ dans le corps, c'était un déterminé

la Dame était tough, mais devant le Doucet elle timbait ${ }^{12}$

il l'avait cassée

un fin connaisseur de ports

un vrai pèlerin

elle l'aimait-il ?

point un mot qu'elle osait, qui se disait

avait peur même de le dire

trop de femmes étiont mortes pour y croire, pour vouloir

tendre les brides de leurs corps

vouloir les laisser venir

mais couchée sur la table, c'était ses mains

qu'elle voulait sentir

c'était le Doucet qu'elle voulait

entendre, $[\ldots]$

ça faisait une semaine qu'elle dormait point

virait, brassait

[...] la biological clock qu'avançait (60-62)

Contrairement à Cécile qui voudrait vivre et écrire le «conte » qu'elle vit avec Jean-Baptiste, Marguerite ne croit pas aux contes de fées et se méfie des histoires qu'on raconte aux femmes pour leur faire accepter le sort qui les attend. Les réflexions de Marguerite montrent bien le risque spécifique que courent les femmes en s'ouvrant aux plaisirs du corps : le risque de tomber enceinte n'est pas présenté comme un problème moral (elle ne craint pas le jugement des autres), mais comme une mise en danger physique, bien réelle pour Marguerite puisque sa propre mère est morte en couches. Dans ce passage, Marguerite se représente ce qui lui apparaît comme le destin des femmes : les grossesses dangereuses, mais aussi l'incertitude de pouvoir compter sur leurs compagnons («tromperies, abandons, vulnérabilité »). Malgré son amour pour le Capitaine 
et son désir d'enfant - qui s'exprime par l'image obsédante d'une petite fille fantôme qui lui tend les bras et que Marguerite voudrait tant sauver -, la Dame hésite à se lancer dans ce nouveau jeu, qui ne lui apparaît pas «fair and square» (33; italiques dans l'original) comme ceux de son casino. Les métaphores utilisées pour montrer sa reddition dans ce passage sont donc plutôt négatives, le désir étant montré comme une perte d'agentivité contrastant avec le caractère décidé de Marguerite : «la Dame était tough, mais devant le Doucet elle timbait / il l'avait cassée ».

Lors du Tyme, soirée où tout bascule, Marguerite accepte finalement de se laisser envahir par son désir pour le Capitaine, désir qui lui traverse le corps (hanche, mains, bras, peau, pieds) :

[...] c'était ça la deal, la feeling, l'entrée

la curve, sa curve, elle

sa hanche, la sienne, son corps

mains, bras, peau pour la guider

la laisser, seule à la camp avec une petite chandelle

p'tit feu, l'eau goutte, les perles

sur le début de sa hanche, qui, ruisseau, elle

la Compound était-y en train de brûler ?

la Dame y pensait même pus, trop concentrée dans ses idées

en train de l'imaginer là, le lilas, le monarch

sentait son arrivée, sa fin proche

des milliers de diamants, glass ceiling fracassé

en train de mouiller, ciel, la place de ses pieds

entendait tcheque part dehors le Piano Man en train de swooner

Le Capitaine havre ${ }^{13}$ ! Le Capitaine havre!

la porte ouverte

la nuit enchantée (« $\mathrm{S}(\mathrm{H}) \mathrm{hhhhhhh} \mathrm{»,} 108$; italiques dans l'original)

Le désir de Marguerite est associé à la renaissance de la nature au printemps (tout en lilas et papillons) ainsi qu'au mouvement sensuel de l'eau qui ruisselle sur son corps et à partir de son corps. L'eau qui baigne Marguerite quand elle pense au Capitaine, est mise en contraste avec le feu de la chandelle dans sa chambre et avec l'incendie qui menace son domaine, mais pas tant pour séparer l'eau du feu que pour les rapprocher : l'eau qui goutte et roule sur la hanche de Marguerite à la fin de la première strophe semble venir du feu même - on peut imaginer les perles de cire se détachant de la chandelle et glissant sur le corps de la femme, qui se retrouve à la fois mouillée et en plein cœur du brasier. Le désir de Marguerite est donc représenté avec des figures paradoxales alliant les contraires, comme une «feeling » de l'extrême. Dans ce cas, la métaphore du plafond de verre fracassé pourrait être comprise non seulement dans le contexte de 
la transgression des rôles genrés en contexte professionnel (elle est, après tout, «la Tycoon » [Grand feu, 11] de Kespukwitk), mais aussi comme l'éclatement de la façade de verre qui tient Marguerite hors du désir et de l'amour, c'est-à-dire à l'écart de son corps. Avec son «plongeon ${ }^{14}$ lors du Tyme, Marguerite fait éclater le verre, ouvre la porte et sort dans « la nuit enchantée », où elle pourra rejoindre le Capitaine.

Bien qu'elle se laisse porter plus facilement par le désir, Cécile parcourt elle aussi un chemin narratif qui l'amène à embrasser le désir avec plus d'assurance. Après son plongeon,

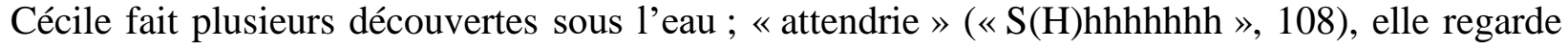
Marguerite s'ouvrir au désir dans la scène précédente puis constate, stupéfaite, qu'au lieu de se noyer, elle est en fait capable de nager. L'eau, l'inconnu, l'écriture, le désir, elle peut s'y mouvoir et les traverser sans mourir; elle est devenue une «sirène astheure » (109), figure ambiguë, traversant les mondes et chantant le désir. En plongeant, Cécile fait la connaissance de son corps - et plus précisément de son sexe :

Cécile nageait !

l'eau rattlait comme le derrière d'une car, lane change, plus creux

elle la sirène astheure

en plongeant, jusqu'au sixième sens, le champignon

le goût du sel, la Cave de son ventre à elle, le silex, le couteau

pour couper la corde, in utero

dans la grotte, sa grotte à elle, trouvée par les scientifiques

voleurs, leurs mains, bras, jambes à grimper

son or, son argent, à la nommer

à prendre du sacré pour expliquer et commenter

une façon, LA direction

elle quittait ça astheure, elle pouvait se rendre plus loin

plus loin

jusqu'à l'oreille de la Dame (109; majuscules dans l'original)

Submergée, Cécile explore son sexe dont elle avait été tenue à l'écart par les voix extérieures (les scientifiques, les médecins, les religieux) qui se l'étaient approprié par leur discours de savoirpouvoir $^{15}$ : non contents d'avoir volé sa richesse, ils avaient aussi contraint sa sexualité à se conformer à une «direction » unique, c'est-à-dire à s'exprimer d'une certaine façon. Reconnaître son désir et son plaisir (et leur donner la «direction » qu'elle veut) équivaut pour Cécile à une renaissance, ce qui est figuré par la corde (le cordon ombilical) qu'elle coupe in utero : elle naît à elle-même en se détachant de ce qui la liait à un rôle préconçu de la femme ainsi qu'à un savoir dogmatique sur elle. L'eau agit donc comme un liquide amniotique qui couve la jeune femme 
pendant sa métamorphose. Ces retrouvailles avec soi permettent à Cécile d'avancer, mais aussi de faire avancer les autres, puisque la fin du passage laisse entendre que Cécile communiquera sa « bonne nouvelle » à Marguerite, la liberté du désir de l'une venant appuyer celle de l'autre.

\section{Mobilité, liberté et création}

Juste avant que Cécile ne se mette à courir vers le lac, on dit que «c'était elle qui devait maintenant décider / sa propre naissance » (Grand feu, 77-78), soulignant le rôle de la prise de risque dans la formation de sa nouvelle identité de poète. En s'arrachant au carrosse où l'accompagnait son oncle, Cécile choisit de devenir une femme libre plutôt que de suivre la voie dictée par les convenances et le pouvoir patriarcal (il est maintes fois souligné que Joachim agit au nom du père mort). Elle sait très bien qu'une fois dehors rien ni personne ne la retiendra : «Cécile décidée / c'était elle l'auteure de son histoire / elle allait plonger 》 (79; italiques dans l'original). Dans la nouvelle comme dans le roman poétique, les images associées à Cécile sont des plus dynamiques et insistent sur sa mobilité et l'exercice de sa liberté. Dès l'ouverture de « $\mathrm{S}(\mathrm{H})$ hhhhhhh », la jeune femme est décrite comme impatiente et prête à tout risquer pour s'accomplir : elle «n'en peut plus. Elle plongera dans le Grand Lac même si ce sera plonger vers sa propre mort » (103). Sa rencontre avec son destin est présentée comme une course où la peur et la sueur s'évaporent (105) puis comme un vol, où son corps est projeté en avant comme une flèche ou un arc-en-ciel :

Cécile volait astheure, une seconde son corps, l'arc-en-ciel, la grâce en train de charger la vitre du sablier en train de craquer

Cécile tranchait l'air, comme tirée l'arc d'un rituel en train de sonner l'écho, résonance

à trembler (105)

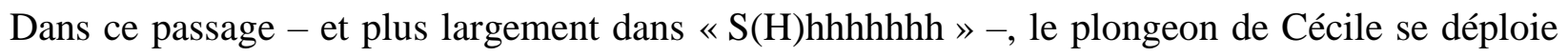
au ralenti : un geste de quelques secondes s'étale sur une dizaine de pages, sa description entrecoupée des sensations physiques ressenties par Cécile, de son excitation et de son état d'esprit fiévreux, de tout ce qu'elle risque en se lançant dans l'inconnu, et de diverses visions qui se présentent à elle. En ralentissant de la sorte l'envolée de Cécile, LeBlanc souligne l'importance de son geste et comment celui-ci implique tout son corps et tout son esprit. Rappelons que LeBlanc a expliqué que «sa » Cécile Murat était inspirée de sa propre expérience 
de devenir écrivaine (LeBlanc à Mousseau) : la peur, le désir et le plaisir de plonger dans l'inconnu et de franchir une frontière invisible qui nous redéfinira. « $\mathrm{S}(\mathrm{H}) \mathrm{hhhhhhh} »$ capte ainsi le poids du saut vers la création en ralentissant la course et en la ramenant à une pellicule qui se déroule image par image ${ }^{16}$.

Comme elle l'indique dans les remerciements en clôture du Grand feu, LeBlanc s'inspire d'événements historiques tel que l'incendie qui a détruit une partie de la Baie Sainte-Marie au dix-neuvième siècle pour « raconter une autre histoire » (89; italiques dans l'original) ; en fait, ce qui l'intéresse n'est pas l'histoire en elle-même, mais plutôt la façon dont le passé fait signe au présent. Par conséquent, LeBlanc ne se laisse pas contraindre par la vraisemblance historique, ce qu'elle indique dans l'image de «la vitre du sablier en train de craquer » («S $(H) h h h h h h h »$, 105) précédemment citée, puis dans celle de la jeune femme qui «traversait les layers de l'histoire » (109) accompagnant la description du plongeon de Cécile. Ce trait était déjà apparent dans le prologue à Alma, où LeBlanc décrit la brume en la rapprochant de «pixels » (9) malgré le contexte historique qu'elle est en train d'établir ${ }^{17}$. Dès ses tout débuts, l'œuvre de LeBlanc se plait donc à brouiller les repères, utilisant les ruptures temporelles pour créer des ponts entre des époques qui se trouvent de la sorte repliées l'une sur l'autre ${ }^{18}$. C'est encore le cas dans Le grand feu et dans « $\mathrm{S}(\mathrm{H}) \mathrm{hhhhhhh}$ », où l'on quitte abruptement le dix-neuvième siècle pour mettre des caméras et un système d'alarme autour de sa propriété (Grand feu, 30), ou encore pour se retrouver en plein film western ou en train d'avaler les kilomètres dans une voiture décapotable :

Cécile était sous l'eau

à Montana, sur le grand chemin regardait les ranchers en train de wrangler ça dans un parc dans le movie, elle était gone à Boston gone en Louisiane dans la Cadillac, top down, ciel rouvert pour rencontrer une femme de Meteghan qu'est point là c'était du Party Mix partout c'était le drive-thru best fried chicken north of 1-10 Screamin' Jay, le tape player, la grosse find au Weymouth Variety Born on the Bayou su rip c'était New York, c'était le désert (« S(H)hhhhhhh », 106)

Dans cette vision, les temps et les espaces se télescopent et Cécile est propulsée dans un lieu et une époque marqués par la mobilité ; ce qu'elle désire, c'est le grisement de la vitesse et de la liberté de mouvement. «Être gone » est son désir le plus profond parce que c'est seulement ainsi qu'elle peut se trouver, se choisir, en se mesurant à l'inconnu. Avec son «ciel rouvert», 
l'espace-temps de la modernité américaine est perçu comme l'endroit et le temps de tous les possibles. C'est en ouvrant la temporalité et l'espace à ses héroïnes que LeBlanc peut raconter son «autre histoire» : plutôt qu'un suicide (103), le plongeon de Cécile dans le lac peut être réécrit comme une plongée en avant où elle circule librement dans une autre incarnation.

Mais «être gone » ne vient pas sans risque et la mobilité est présentée comme un risque qui coûte cher, puisque l'accès au lac (à la création, au désir, à la réinvention de soi) est freiné par la présence d'un «dragon». Le Dragon ${ }^{19}$ qui se cache peut-être dans le lac devrait en premier lieu menacer Cécile, puisque c'est elle qui plonge à sa rencontre; toutefois, dans «S(H)hhhhhhh », c'est Marguerite qui fait face au Dragon :

la Dame était là itou [en-dessous de l'eau], Cécile la voyait dans la light mais Marguerite Theriault voulait pus l'histoire Cécile la voyait, prise dans sa propre chambre noire dans le cœur de sa maze, devant le monstre, le grand Dragon tant redouté c'était point une bête, c'était une feeling, c'était perdre son idée [...] deux bras masqués l'aviont prise, sans mots c'était un Tyme, c'était un full-blown fiasco (107; italiques dans l'original)

Ce passage décrit la menace comme quelque chose d'abstrait, « une feeling », ce qui rappelle l'Antercri d'Alma, lui aussi une menace qui «s'attrape point $»^{20}(11)$. Si le Dragon «c'était perdre son idée » et tomber dans « un full-blown fiasco », on pourrait considérer que Marguerite est aussi en lien avec l'écriture et la création ${ }^{21}$, comme le double inversé de Cécile. La chambre noire, le lieu de l'écriture, le cœur du labyrinthe, est l'endroit où elle doit plonger au risque de tomber face à face avec l'empêchement à l'écriture : le doute, perdre son idée, ne plus voir ni vouloir l'histoire. Assez loin de la représentation idyllique du rapport aux mots d'Alma qui sautait d'un mot à l'autre comme l'on traverse une rivière en appuyant son pied sur les roches sûres, sans glisser ${ }^{22}$, le rapport de Cécile et de Marguerite à l'écriture et à la création est empreint de tourments et de doute : l'œuvre est-elle valide, ou sera-t-elle un «full-blown fiasco »? Le Tyme organisé par Marguerite le jour de la Sainte-Cécile, patronne des poètes et musiciens, est un moment déterminant parce qu'à même son chaos, son grand feu, une (ou deux ?) poète fera le grand saut et embrassera la création ${ }^{23}$.

Dans Le grand feu, la venue à l'écriture de Cécile est pétrie d'angoisse, ce qui est canalisé dans son rapport aux histoires étranges qui circulent au sujet de la Mary Celeste, un bateau fantôme qu'on a retrouvé en mer sans savoir ce qui était arrivé à son équipage. Cette histoire captive Cécile par son mystère : «rinque une histoire, peut-être / peut-être, mais Cécile était pris 
/ l'histoire y collait partout dans le tchœur / dans son esprit (23). L'obsession de Cécile pour les récits de tout un chacun tentant d'expliquer les aventures du bateau la pousse à une écriture fiévreuse, qui tente de répondre à la commande de Marguerite pour des «anecdotes colorées » (23) récoltées des récits oraux échangés à la Cantine. Toutefois, son écriture est sans cesse empêchée par le surgissement de la Sirène (24), celle qui aurait pris la vie de son père lors d'un naufrage. L'écriture de Cécile est de la sorte sans cesse dévoyée de l'anecdote du bateau perdu en mer pour être ramenée à l'histoire personnelle de la mort de son père et à celle de son propre départ imminent pour l'Europe.

La Sirène est une autre figure joignant l'eau et le feu, à la suite du Dragon gardien du lac, puisqu'elle apparaît à Cécile dans la flamme de la bougie à la lumière de laquelle elle écrit dans sa chambre :
chaque fois que Cécile allumait sa petite chandelle pour écrire faire avancer le travail demandé la Sirène apparaissait dans la flamme, drôle de génie [...] arrêtait à mi-chemin, l'idée originelle la Sirène prenait la voix de son père c'est elle qui l'avait, qui le tchenait amarré sous l'eau, dans les bras de la grande mer salée [...] Cécile commençait, recommençait page après page, un début de quoi ? pouvait même pas décrire la rime des images faire suite de ses idées
[...] un conte ? rien de ce qu'elle écrivait ressemblait à ce qu'on lui avait demandé rien de ce qu'elle avait appris à l'Académie structures, charpentes des lettres, tout dégringolait sous ses doigts, ses mains à trébucher (24 ; italiques dans l'original)

Si Cécile connaît la norme de l'Académie, il lui est impossible d'écrire tant qu'elle se croit obligée d'y obéir. De même, Cécile comprend que Marguerite aimerait lire des histoires populaires retranscrites de l'oral, mais elle n'est pas non plus capable de répondre à cette commande de folklorisme. Pour devenir poète, Cécile doit accepter de braver l'inconnu, de suivre la Sirène qui la mène peut-être vers la mort (à la suite de son père), mais qui la mène en tout cas dans une voie personnelle qui est présentée comme la seule voie possible. Remarquons que dans « $\mathrm{S}(\mathrm{H})$ hhhhhhh » Cécile était elle-même devenue une sirène, trouvant une nouvelle 
façon de respirer et de se mouvoir sous l'eau, de se rapprocher de l'épave et de la création, tandis que dans Le grand feu, la Sirène est une apparition, un génie, une force extérieure qui l'attire, mais dont Cécile ne sait si elle lui sera bénéfique ou maléfique.

Coincée dans un carrosse avec son oncle Joachim sur la route pour le Tyme qui lui paraît interminable, Cécile se sent mal à l'aise. La «boite noire » (49) du carrosse est l'espace-temps où se prépare sa «sortie du placard » en tant que poète, ce qui la rend malade d'angoisse ; tout lui est familier et pourtant tout lui semble étranger et vaguement menaçant :

dans la boite noire du carrosse

un frette lui montait le long de l'échine

son ventre était toute brassé

elle avait de la misère à bien se placer

connaissait bien le banc, le coussin du carrosse

avait plus d'une fois accompagné Casimir et Marie [ses parents adoptifs]

en promenade sur le Chemin du Roi

mais tout d'un coup, tout

était transformé, comme étranger

vire pis brasse, vire pis brasse

ça arrêtait point, ça montait, ça dévalait (49)

À mon sens, la «boite noire » du carrosse est un endroit ambigu où se prépare l'écriture : Cécile est malade de trac, juste avant sa métamorphose en poète. Elle connaît pourtant les mots, le décor lui est familier, mais de soudainement passer de vivre cette réalité à l'écrire chamboule tout : le connu devient inconnu, et tout semble neuf et mystérieux sous son nouveau regard. La jeune femme est convaincue qu'elle doit sacrifier sa vie pour faire entendre sa voix :

Sainte-Cécile morte

Sainte-Cécile gone pour croire

oser vouloir entendre une autre parole chanter

vouloir trouver harmonie, laisser parler

soleil, vibrations, l'originel (52)

Présenté de cette façon, son projet littéraire se place à la fois dans l'ouverture à une voix singulière et personnelle («entendre une autre parole chanter»), et dans la représentation adéquate de son environnement, de ce qui est autour d'elle (« laisser parler / soleil, vibrations, l'originel »). L'on retrouve ici les deux axes de la poétique de LeBlanc : l'importance de la mise en scène de la subjectivité - qui passe par l'incarnation (au sens d'embodiment) dans des corps sensibles et sensuels - et la quasi transparence des corps des conteuses, conteurs, poètes et musiciens, qui s'effacent devant la Voix qui raconte la vie dans l'espace propre à la Baie (ou au 
village cadien dans Amédé). LeBlanc positionne sa voix d'écrivaine sur ce point fragile entre l'individuel et le collectif, l'historique et le contemporain ${ }^{24}$.

« $\mathrm{S}(\mathrm{H}) \mathrm{hhhhhhh} »$ se termine sur deux culminations : Marguerite qui fait l'amour avec le Capitaine et Cécile qui rejoint la Mary Celeste sous l'eau. Quand elle touche presque au bateau, l'espace encore une fois s'ouvre devant elle :

elle nageait

[...] voyait la Mary Celeste, le naufrage, là, au fond de l'eau

l'échine de la mer, couchée dans le berceau

elle était là, Cécile la voyait, la ghost ship elle-même

[...] elle pouvait presque la toucher

pouvait se rendre plus loin astheure

au fond, au fi fin fond du lac iousque la sirène l'avait guidée

la racine, la matrice

rendue au champ du possible (110)

Plonger était difficile, terrifiant, mais une fois engloutie, Cécile est sortie de la peur, « rendue au champ du possible », avançant vers le « fi fin fond » des choses en explorant son univers. Cette exploration prendra la forme d'un livre aux pages blanches qui l'attire irrésistiblement :

le livre

les pages blanches qu'elle avait tant souhaitées

les pages blanches de l'idée

le livre était là sur la table à cuisine de la Mary Celeste

dans le cœur de l'épave la seule affaire à garder

[...] le livre était là, la guettait, posé

le livre comme une note parée à sonner

le livre comme le début d'une neuve histoire à tracer (111)

Le livre attend la poète, «par[é] à sonner » maintenant qu'elle a fait cette route vers lui, qu'elle a risqué sa vie pour entendre « la bass note de la tune » (111) qui vibrait sous l'eau.

$* * * * * * * * * * * * * *$

Tous les romans poétiques de LeBlanc publiés à ce jour mettent en scène des créatrices ou des créateurs : une conteuse (Alma) et des conteurs (Prudent et Célestin Trahan); des musiciens (Amédé et Lejeune) ; une poète en herbe (Cécile). Pour chacun de ces personnages, la création s'inscrit profondément dans le corps : leur voix, leur musique et leurs cris sourdent de leur corps et de la terre (acadienne ou cadienne) dans laquelle elles et ils s'ancrent. Dans le cas de Cécile, la création emplit d'abord ses bras puis l'ensemble de son corps qui se réveille ( $\mathrm{S}(\mathrm{H}) \mathrm{hhhhhhh} »$, 104) et plonge ; alors qu'Alma était associée au territoire surtout à travers des métaphores liées à la terre et aux plantes qui y poussent ${ }^{25}$, pour Cécile l'enracinement passe par le rapport à l'eau - 
elle plonge, nage, pagaye, fait du portage, et « sui[t] la rivière, les veines de la terre » (104). De terre, de racines et d'eau, tels semblent être les personnages féminins de la Baie dans l'œuvre de LeBlanc; cependant, tout en maintenant leurs attaches dans le territoire ${ }^{26}$, les femmes de LeBlanc sont représentées comme jouissant de leur liberté dans leur expérience du désir, de la création et de la mobilité. Avec Cécile et Marguerite, LeBlanc crée des personnages qui plongent dans le désir et la création sans naïveté, les yeux ouverts, dans la peur et la gravité, conscientes que la mort les attend peut-être; mais elles le font aussi dans la joie du risque et l'ouverture au changement, qui fait que l'univers romanesque de LeBlanc n'est jamais sclérosé.

Le grand feu présente Kespukwitk comme un espace métissé - probablement le plus métissé de l'œuvre de LeBlanc jusqu'à ce jour -, peuplé principalement d'Acadiennes ou d'Acadiens revenus du grand voyage telle Marguerite, mais où l'héritage mi'kmaq est toujours visible et qui attire des visiteurs franco-américains (un «jeune dandy de la Philadelphie » [Grand feu, 11]), canadiens-français (les «journalistes du Mont Royal» [69]) et européens (Joachim Murat). Cette communauté hétérogène s'ouvre à ce qu'Élise Lepage nomme «la version minimale du Nous » (50; italiques dans l'original), créant une ouverture à l'autre, qui qu'il soit, sans pour autant renvoyer à un projet collectif ou à une communauté spécifique. Bien ancrée dans la Baie, LeBlanc veut embrasser le monde et ses personnages sont en constant mouvement - rappelons que Le grand feu décrit des courses, des promenades à cheval et en carrosse, des traversées en bateau, un plongeon et un parcours à la nage. Nous pourrions rapprocher la poétique de LeBlanc à la «poéthique » que Lepage, reprenant un néologisme de Jean-Claude Pinson, repère chez la poète franco-ontarienne Andrée Lacelle : il s'agirait, pour LeBlanc comme pour Lacelle, de «construire une subjectivité [...] tout en ménageant une place à l'autre » (Lepage 57). Quand Marguerite décrit le Tyme dont elle rêve, elle met en place une série de contrastes : "faut recréer de quoi de wild, de quoi de fou, mais contrôlé / de quoi de vieux, de quoi de neuf, de quoi de juste assez / faut que ça sente l'original, l'inspiration / rien d'inventé (Grand feu, 40 ; italiques dans l'original). Les oppositions permettent de placer la fête parfaite dans l'équilibre presque impossible (puisque le full-blown fiasco guette toujours) entre le fou et le sensé, l'ancien et le moderne. À mon sens, LeBlanc tient un peu le même pari, ancrant ses œuvres poétiques - surtout les deux plus récentes, Le grand feu et « $\mathrm{S}(\mathrm{H}) \mathrm{hhhhhhh} »-$ dans la délicate position mitoyenne entre l'obscurité du sens et la clarté, l'histoire et la modernité, le collectif et le particulier. 


\section{Bibliographie}

Bourcier, Marie-Hélène. «Post-pornographie ». Dictionnaire de la pornographie. Dir. Philippe di Folco. Paris : PUF, 2005. 378-380.

Delorme, Julie. «Les lieux du feu dans l'œuvre romanesque de Daniel Castillo Durante ». La littérature franco-ontarienne depuis 1996 : Nouveaux enjeux esthétiques. Dir. Lucie Hotte et François Ouellet. Sudbury : Prise de parole (Agora), 2016. 235-254.

Doyon-Gosselin, Benoit et Jean Morency. «Le monde de Moncton, Moncton ville du monde : l'inscription de la ville dans les romans récents de France Daigle ». Voix \& Images 29.3 (2004) : 69-83.

LeBlanc, Georgette. Alma. Moncton : Perce-neige, 2006.

---. Amédé. Moncton : Perce-neige, 2010.

---. Le grand feu. Moncton : Perce-neige, 2016.

---. Prudent. Moncton : Perce-neige, 2013.

---. « S(H)hhhhhhh ». Estuaire 160 (2015) : 101-111.

Lepage, Élise. «L'accueil, la confiance dans l'œuvre d'Andrée Lacelle ». La littérature francoontarienne depuis 1996: Nouveaux enjeux esthétiques. Dir. Lucie Hotte et François Ouellet. Sudbury : Prise de parole (Agora), 2016. 39-61.

Mousseau, Sylvie. «Georgette LeBlanc lance son $4^{\mathrm{e}}$ roman poétique ». L'Acadie nouvelle 25 avril 2016. http://www.acadienouvelle.com/arts-et-spectacles/2016/04/25/georgetteleblanc-lance-4e-roman-poetique/?pgnc=1 (consulté le 20 mai 2016).

Valcke, Juliette. «De Roland à Amédé : Filiation médiévale d'un roman acadien du $21^{\mathrm{e}}$ siècle ». Littérature acadienne du $21^{e}$ siècle. Dir. Cécilia W. Francis et Robert Viau, Moncton : Perce-neige (Archipel / APLAQA), 2016. 17-30.

\section{NOTES}

${ }^{1}$ À l'automne 2015, Georgette LeBlanc a confié à l'auteure que le titre de la nouvelle, «S(H)hhhhhhh », faisait référence au bruit d'un corps qui entre dans l'eau. Notons au passage que LeBlanc orthographie le nom de famille de Marguerite légèrement différemment dans les deux œuvres - «Theriault » dans « $\mathrm{S}(\mathrm{H}) \mathrm{hhhhhhh}$ » et «Thériault » dans Le grand feu -, mais il s'agit du même personnage. Une même variation se repère dans le nom du bateau, Mary Celeste ou Mary Céleste.

${ }^{2}$ Le domaine de Marguerite, spacieux et isolé, est surnommé « la Compound ».

${ }^{3}$ D'ailleurs, puisque « Kespukwitk » est le nom de la région mi'kmaq où est située la Baie Sainte-Marie, il va sans dire que Kespukwitk existait bien avant l'arrivée de tous les Européens et leurs descendants, depuis les « temps premiers » (Grand feu, 15). Dans Le grand feu, LeBlanc inscrit cet espace dans plusieurs réseaux simultanément : le 
nom mi'kmaq le marque comme territoire autochtone, tandis que la plupart des personnages principaux et la langue de la narration et des dialogues le placent comme pleinement acadien. En outre, Kespukwitk est présenté comme « le Sud-Ouest du Far-Est » (11), un clin d'œil humoristique au «Far West » et à l'imaginaire américain qui y est associé. Notons enfin que Marguerite vient elle-même des États-Unis ; pour elle, l'Acadie est un espace retrouvé, regagné (dans un casino !) après l'exil et l'errance causés par la Déportation.

${ }^{4}$ Je renvoie ici obliquement aux travaux de Benoit Doyon-Gosselin et Jean Morency qui ont montré comment France Daigle plaçait Moncton comme «centre du monde» dans ses romans à partir de 1953, chronique d'une naissance annoncée. Doyon-Gosselin et Morency (76) identifient dans Pas pire deux stratégies employées par Daigle pour sortir les habitants de Moncton «de leur enfermement identitaire » : 1. «transformer les notions de centre et de périphérie, c'est-à-dire faire de Moncton sinon le centre du monde, du moins un des centres du monde, en vertu du principe qu'il existe une infinité de centres du monde »; 2. «mettre en relation cette ville à la fois quelconque et unique avec le reste du monde ». Ils concluent qu' «[u]tilisées de concert, ces deux stratégies contribuent à faire de Moncton une ville transcendant ses propres limites, une ville centrée et décentrée en même temps, une ville se suffisant à elle-même tout en étant reliée à d'autres villes, à d'autres espaces, à d'autres lieux, ce qui débouche sur un bouleversement radical de la conception traditionnelle du centre et de la périphérie » (76). De même, dans l'œuvre de LeBlanc, la Baie Sainte-Marie est établie comme centre du monde tout aussi valable que n'importe quel autre et est mise en relation avec une variété d'autres espaces plutôt que d'être représentée comme un endroit isolé et homogène. Dans Amédé, l'intrigue se déplace vers un village cadien en Louisiane, mais la dynamique centre-périphérie leblancienne demeure inchangée.

${ }^{5}$ J'ai étudié la subjectivité féminine dans Alma dans un article à paraître à l'hiver 2017 dans la Revue de l'Université de Moncton, «Les quatre saisons d'Alma : Une lecture écoféministe d'Alma de Georgette LeBlanc ».

${ }^{6}$ Attendre.

${ }^{7}$ Joachim Murat est un personnage historique ; militaire français marié à la sœur de Napoléon, Caroline Bonaparte, il sera nommé par celui-ci « Roi de Naples » en reconnaissance de son service.

${ }^{8}$ Le personnage de Joachim s'exprime en français standard européen et est souvent dérouté par les usages acadiens, qu'il perçoit comme des marques d'infériorité issues de l'éloignement régional et du brouillage des classes sociales visibles à Kespukwitk.

${ }^{9}$ Le fait que son débat intérieur sur le désir et la maternité se déploie pendant que la Dame se fait masser me semble intéressant : son angoisse, comme son désir, s'exprime dans son corps (ses années de refus lui ont fait des nœuds dans le dos).

${ }^{10}$ Nœuds.

11 Travail.

12 Tombait.

${ }^{13}$ Arrive.

${ }^{14}$ Dans la nouvelle comme dans le roman poétique, c'est Cécile et non Marguerite qui plonge dans le lac ; toutefois, Marguerite plonge elle aussi de façon figurée puisqu'elle se laisse emporter par le désir, ce qu'elle avait refusé de faire jusque-là.

${ }^{15}$ La chercheure queer Marie-Hélène Bourcier a montré comment la pornographie standard, par exemple, émanait d'un vaste champ de savoir-pouvoir moderne qui pose un «expert» masculin (médecin, psychanalyste, pornographe) en spécialiste d'une nature féminine sexuelle à expliquer. Dans ce qu'elle nomme la «postpornographie », les minorités sexuelles reprennent ce discours sur elles et eux et le renversent, prenant les rênes de leur représentation. Un phénomène semblable s'observe dans une partie de la littérature des femmes contemporaine, avec des écrivaines aussi diverses que Wendy Delorme, Pattie O’Green, Virginie Despentes, Chloé Savoie-Bernard, Annie Ernaux, Nelly Arcan ou Natasha Kanapé Fontaine.

${ }^{16}$ Notons que le numéro d'Estuaire dans lequel est paru « $\mathrm{S}(\mathrm{H})$ hhhhhhh » avait comme thème « Cinéma de poésie ». La nouvelle poétique de LeBlanc est particulièrement visuelle et joue sur la mobilité spatiale et temporelle, comme nous le verrons plus loin.

${ }^{17}$ Alma s'ouvre à l'aube du vingtième siècle.

${ }^{18}$ Pour une étude du rapport entre le temps de l'histoire et le temps vécu dans les quatre romans poétiques de LeBlanc, on consultera avec profit l'article de Julie St-Laurent dans le présent numéro.

${ }^{19}$ Le Dragon, animal mythique crachant du feu, est ici double - comme la sirène, sur laquelle nous reviendrons plus loin -, puisqu'il est associé à la fois à l'eau du lac qu'il garde, et au feu qui lui permet de défendre ce lieu sacré. Julie Delorme note la nature paradoxale du feu, qu'on recherche parce qu'il nous procure sécurité et réconfort (lumière et chaleur), mais qui est aussi ce «qu'il ne faut surtout pas toucher au risque de nous brûler » (236; 
italiques dans l'original). Le dragon représente ces deux côtés : à la fois menace et protection, selon la position de chaque individu.

${ }^{20}$ Dans Alma, la menace qui guette les gens sur la côte ne s'incarne pas dans un homme, mais demeure au contraire diffuse comme la brume, c'est-à-dire un peu partout et nulle part en particulier : «François le Premier guettait un homme grand / déjà fait déjà mâle / mais l'Antercri c'était point un homme / c'était même point tout à fait un rêve / l'Antercri c'était la promesse / c'était le braquement du désir / de vouloir attraper de-quoi qui s'attrape point » (11). Au sein du roman, on pourrait dire que le compagnon d'Alma, Pierrot, a succombé au chant enjôleur de l'Antercri en projetant ses désirs sur la modernité américaine, ce qui brouille sa vue et l'empêche d'apprécier ce (et celle) qu'il a sous les yeux. Voir à ce sujet mon article à paraître sur Alma dans la Revue de l'Université de Moncton.

${ }^{21}$ D'ailleurs, dans Le grand feu, Marguerite incite Cécile à écrire en lui commandant des pages sur les histoires populaires que les femmes échangent dans les cuisines (23).

${ }^{22}$ Le poème «la compernure » illustre le talent d'Alma avec les mots (à la fois pour la lecture et l'écriture) en la contrastant à sa sœur, qui n'a pas hérité du même don : «ma sœur a de la misère à lire / mais moi ej trouve ça comme une game / j'essaie d'i expliquer : / c'est comme des roches dans la rivière / tu fais rinque de suivre la phrase comme tu suis les roches / [...] faut rinque faire confiance aux mots / comme qu'on fait confiance aux roches » (47; italiques dans l'original).

${ }^{23}$ Puisque la tradition annuelle à Kespukwitk est que les poètes et les musiciens plongent dans le lac le jour de la Sainte-Cécile (Grand feu, 28), c'est une acceptation et une revendication de son rôle de poète pour Cécile que de plonger elle aussi cette année-là.

${ }^{24}$ Juliette Valcke a par ailleurs montré comment LeBlanc s'inspirait de la forme et du style propres aux œuvres médiévales (notamment la chanson de geste) pour créer des romans poétiques empreints d'oralité qui font entrer la « petite histoire » cadienne et acadienne dans « une histoire littéraire beaucoup plus vaste » (17), et qui recyclent des modes anciens et étrangers afin de mettre en mots le local et le moderne.

${ }_{25}$ J'ai analysé ces images dans mon article sur Alma à paraître dans la Revue de l'Université de Moncton.

${ }^{26}$ Notons que ces attaches au territoire passent également par un usage sensuel et évocateur de la langue de la Baie. 